\title{
Dawn of a New Age Fissure Sealant? A Study Evaluating the Clinical Performance of Embrace WetBond and ART Sealants: Results from a Randomized Controlled Clinical Trial
}

\author{
Praveen Bhoopathi Haricharan ${ }^{1}$ Naveen Barad ${ }^{2}$ Chetan R. Patil ${ }^{3}$ Sreenivas Voruganti ${ }^{4}$ \\ Durga Prasad Mudrakola' Neeraja Turagam
}

1 Faculty of Dentistry, AIMST University, Malaysia

${ }^{2}$ Department of Conservative Dentistry and Endodontics, Nanded Rural Dental College \& Research Center, Nanded, Maharashtra, India

${ }^{3}$ Department of Conservative Dentistry and Endodontics, Maratha Mandal Dental College, Belgaum, Karnataka, India

${ }^{4}$ Department of Community Dentistry, Kamineni Institute of Dental Sciences, Nalgonda district, Telangana, India

\begin{abstract}
Address for correspondence Praveen Bhoopathi Haricharan, MDS, Dental Public Health Unit, Faculty of Dentistry, AIMST University, Semeling, Bedong, Kedah, Malaysia 08100 (e-mail: drprav1983.dentist@gmail.com).
\end{abstract}

Eur J Dent 2019;13:503-509

\begin{abstract}
Keywords

- clinical trial

- fissure sealants

- preventive dentistry

- dental caries

- ART sealant (atraumatic restorative treatment)

- retention

- Embrace WetBond
\end{abstract}

Objectives The main purpose of this article is to evaluate the clinical performance of atraumatic restorative treatment (ART) sealant versus Embrace WetBond sealant in terms of retention and fissure caries prevention among a section of school children in the southern Indian state of Karnataka (ClinicalTrials.gov NCT02716558).

Materials and Methods After obtaining consent from the parents and screening the children against the inclusion cum exclusion criteria, 90 school children whose mandibular first molars were caries free and with a well-defined pit and fissure system were recruited for this spilt mouth trial. The respective molars were allocated either to the ART sealant or the embrace group after the randomization process. The sealants were applied according to the manufacturer's instructions and followed up at the end of 3rd, 6th, and 12th month, respectively. The outcome measures assessed were the retention and the caries preventive effects of the materials.

Results At the end of the 6th month, about one-fourth of the sealants in either group remained totally intact, without evidence of caries. No significant differences were seen between the sealants either in terms of retention or caries preventive benefits at the end of 12 months.

Statistical Analysis The chi-squared test was used to check differences in proportions. The significance value was set at $<0.05$. Kappa test was performed to assess the intraexaminer reproducibility with respect to retention and caries status.

Conclusion The moisture-tolerant resin sealant could not replicate the physical properties usually associated with conventional resin sealants. The usage of ART sealants was deemed to be less cumbersome in an outreach setting as observed in this trial.

\section{Introduction}

Dental caries is a multifactorial disease of the oral cavity caused by an alteration in the composition of the bacterial plaque, leading to an imbalance between the demineralization and remineralization cycle, which clinically manifests as a cavitated/ noncavitated carious lesion. ${ }^{1,2}$

The comparison between the National Health and Nutrition Examination Survey data reported for the phases between 1999 and 2004 and 2011 and 2012 revealed that 
the prevalence of dental caries has declined and this waning was not been uniform across age groups, sociodemographic status, and the tooth surface sites. ${ }^{3,4}$

Further analysis of the susceptibility on tooth surface sites indicated that the drop was observed more in the smooth surfaces than on the occlusal surfaces. ${ }^{5,6}$ The occlusal surfaces of the teeth are particularly prone to decay primarily due to the anatomical nature of these surfaces that favor plaque retention. ${ }^{7.8}$

Currently, the use of pit and fissure sealants is considered as a highly effective tool for preventing dental caries on the occlusal surfaces of the teeth and has been used in the dental profession since the 1960s. s,9,10 $^{6}$ The underlying concept of placing fissure sealants is to create a physical barrier and thus cutting off the nutritional supply to the bacteria. ${ }^{11}$ Over the years, two classes of sealants have emerged and these are the resin and glass ionomer-based sealants and are recommended for children placed in the high-caries-risk category. ${ }^{6}$

Resin-based sealants by far have been the most effective in preventing occlusal carious lesions as demonstrated in many clinical trials. ${ }^{12}$ However, these sealants have hydrophobic properties that demand a near total moisture control which often limits its clinical applications. ${ }^{10}$ An advanced resin-based sealant (Embrace WetBond sealant) has been developed that incorporates di-tri and multifunctional acidic acrylate monomers with a practically designed hydrophilichydrophobic balance. The resultant product is a resin sealant that has hydrophilic properties and sets even in the presence of moisture. ${ }^{13}$

Glass ionomer sealants being hydrophilic in nature are used in situations where profound moisture control is difficult to attain. Nonetheless, these sealants too have their share of issues such as low abrasive strength, high solubility in oral fluids and, thus, have a lower retention rate in comparison to the resin-based sealants. ${ }^{14,15}$ Highly viscous glass ionomer cements have been introduced for application on the caries-susceptible pit and fissures by the press finger technique borrowed from the atraumatic restorative technique (ART). ${ }^{16,17}$ These sealants have improved physical properties such as reduced moisture sensitivity, lower solubility, and better retention rate as compared to the conventional glass ionomer sealants. ${ }^{18}$

Embrace WetBond and ART sealants have improved physical properties that entitle them to be placed in clinical situations where in profound moisture control would be a demanding prospect. The caries preventive effects of ART sealants have been similar when pitted against conventional resin-based sealants in spite of lower retention rates. ${ }^{19,20}$ However, mixed results were obtained in the case of Embrace WetBond sealant in comparison to conventional hydrophobic resin sealants. ${ }^{21-25}$

There is a dearth of literature on the relative efficacy of ART and Embrace WetBond sealant in terms of retention and caries preventive benefits. Hence, a comparative study between these hydrophobic materials was considered appropriate. The aim of the present investigation was to compare the retention and caries-preventive effects between ART sealants and Embrace WetBond sealants among a section of school children in one of the districts in southern India. The null hypothesis states no difference between the two materials.

\section{Materials and Methods}

This spilt mouth randomized controlled clinical trial was carried out in Belgaum state of Karnataka located along the northern part of the Western Ghats in India. The study has been registered with the US National Library of Medicine (ClinicalTrials.gov NCT02716558) and ethical clearance was obtained from the institutional review board of Maratha Mandal Nathajirao G. Halgekar Institute of Dental Sciences \& Research Centre (Board Approval Number: 907).

The study was carried according to the guidelines of the Helsinki declaration and follows the CONSORT guidelines. The water supply of the town is not fluoridated and the usage of fluoridated toothpaste was widespread in the community. The study population consisted of children belonging to a low socioeconomic status, in the age group between 7 and 11 years of age enrolled in three government schools in the district of Belgaum.

The two groups of sealants used in this spilt mouth randomized clinical trial are the ART sealants (Ketac-Molar Easy Mix, 3 M ESPE, Seefeld, Germany) and Embrace WetBond (Pulpdent Corporation Watertown, Watertown, Massachusetts, United States).

The children's parents/guardians were explained about the benefits and risks associated with this trial. Only those children, whose parents gave a written informed consent, were clinically examined by a trained dentist. The baseline clinical examination was carried out within the respective school premises by a graduate student. Data were also recorded on the frequency of sugar exposures and the oral hygiene status.

The inclusion criteria were as follows: (1) children with a DMFT $\geq 2$; (2) poor oral hygiene status; (3) fully erupted, noncarious, contralateral mandibular first molars exhibiting a deep or noncoalesced pit and fissure system; (4) history of irregular dental visits and a high frequency of exposure to sugars based on their dietary patterns. A child had to satisfy these criteria in order to be included in the study. The children who meet the inclusion criteria could be at least be classified either into a medium or a high-caries-risk category.

Children with developmental anomalies or with hypoplastic teeth, presence of cavitated carious dentinal lesions on the occlusal surface of the mandibular first molars, and uncooperative children were excluded from the study.

The graduate student who had carried out the baseline clinical examination of the school children was handed the responsibility for placing the sealants and thus was extensively trained on the theoretical and the clinical aspects of pit and fissure sealants.

Critical aspects such as the indications for sealant usage and application procedures were deliberated and reinforced. Prior to sealant applications on the study sample, the graduate student was calibrated on a certain number of children attending the preventive clinics of the dental school in that district. These children were walk-in patients and not a part of the study sample. 


\section{Sample Size Calculation}

Based on the minimum expected difference of $20 \%$ in retention between the two materials at the end of 1 year, $(\alpha=0.05$ and $80 \%$ power) and attrition rate at $30 \%$, the sample size was estimated to be 90 teeth per group.

\section{Randomization}

Randomization was carried out by a toss of coin to allocate the molars into either of the two groups. For example, if the left mandibular molar received a resin sealant, then the corresponding right mandibular molar received the glass ionomer-based sealant.

\section{Clinical Application}

The clinical applications of the sealants were carried out in the school premises by using portable dental chair and equipment that replicated an outfield setting. No electrically driven equipment was used in the application of the sealants.

The occlusal surfaces of the molars in the moisture tolerant resin group were etched with $37 \%$ phosphoric acid for 15 seconds and rinsed with copious amount of water to get rid of the etchant. A cotton pellet was then used to remove excess water on the tooth surface and to not desiccate the surface. The occlusal surface remained slightly moist and glossy prior to the application of the resin sealant and later light cured for 20 seconds following the placement of the sealant.

With regard to the ART sealant application, the occlusal surfaces of the molars were treated with the liquid component of the glass ionomer for 15 to 20 seconds, cleaned, and dried with cotton pellets. The glass ionomer sealant was mixed according to the manufacturer's instructions and placed using the press finger technique. ${ }^{26}$ Appropriate postoperative instructions were given.

\section{Follow-up Evaluations}

The subjects were examined at the end of the 3rd, 6th, and 12th month, respectively, by an independent examiner, who was not involved in the study since its inception. The follow-up clinical examination was performed at the preventive clinics of the dental school, by contacting the parents/guardians and summoning the children in an orderly manner.

Duplicate examinations were carried out at each of the scheduled follow-up intervals on about $10 \%$ of the sample. Separate time was allocated to examine the children who could not be examined during the primary follow-up intervals.

The randomization details were not shared with this examiner and the data sheets filled up at designated time intervals were kept confidential until the end of the study period.

Community Periodontal Index probes and sharp sickleshaped dental explorers were used to assess retention and carious lesions that developed. Resealing of the partially or completely worn-out sealants observed at subsequent follow-ups were not carried out in this trial. The modified Simonsen's criteria $^{27}$ ( $\boldsymbol{-}$ Table $\mathbf{1}$ ) were used to assess the retention and the
Table 1 Modified Simonsen's criteria

\begin{tabular}{|l|l|}
\hline Score 0 & No loss of sealant and no evidence of caries \\
\hline Score 1 & Partial loss of sealant and no evidence of caries \\
\hline Score 2 & Partial loss of sealant and evidence of caries \\
\hline Score 3 & $\begin{array}{l}\text { Complete loss of sealant and no evidence of } \\
\text { caries }\end{array}$ \\
\hline Score 4 & Complete loss of sealant and evidence of caries \\
\hline
\end{tabular}

presence or absence of carious lesions. The cavitated dentin carious lesions were detected as per the diagnostic criteria of the World Health Organization Oral Health Survey. ${ }^{28}$

\section{Statistical Analysis}

The data collected were entered on to the Microsoft Excel Sheet and analysis was performed by SPSS (Statistical Package for Social Sciences) Version 22.0 (IBM Corporation, Chicago, United States). The chi-squared test was used to check differences in proportions. The significance value was set at $<0.05$. Kappa test was performed to assess the intraexaminer reproducibility with respect to retention and caries status.

\section{Results}

A total of 323 school children were screened by matching them up against the inclusion and the exclusion criteria. Out of these, 116 children satisfied the inclusion criteria and 90 of them were randomly picked to be recruited for the clinical trial ( - Fig. 1). Fortunately, for that academic year, there were no dropouts from these schools and thereby, all the children recruited for the trial were examined at the scheduled intervals. The mean age of the children was 9.4 years with $44 \%$ of them being boys and the remaining $64 \%$ of them were girls.

A total of 90 children constituting 180 permanent mandibular molars were followed up at the end of the 3rd, 6th, and 12th month, respectively. The results of the duplicate examinations showed good intraexaminer reproducibility with kappa value of 0.87 for sealant retention and 0.90 for caries status.

There was no significant difference between the two groups of sealants at the end of the 3rd month ( $p=0.673$ ), with about $66 \%$ of the glass ionomer cements (GIC) sealants and $60 \%$ of the resin sealants being completely intact, with no evidence of caries. At the end of the 6th month, about onefourth of the sealants in either group remained totally intact, without evidence of caries. Conversely, about 18 and $11 \%$ of GIC and resin group sealants were completely lost, without any evidence of dental caries. There has been no significant difference between these groups of sealants at the end of 12 months, with the proportion of dentinal caries at 7.8 and $6.7 \%$ for the GIC and resin group respectively ( - Table 2 ).

\section{Discussion}

Pit and fissure sealants have been established as a cornerstone material in preventive dentistry. These widely used 
382 children ( $7-11$ years)

The specifics of the trial explained. Informed consent sought

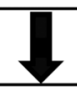

323 children examined after obtaining the informed consent

Examined and matched against inclusion/exclusion criteria

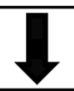

116 children satisfied the inclusion criteria, 90 randomly selected to be a part of the clinical trial ( 90 subjects, 180 molars)

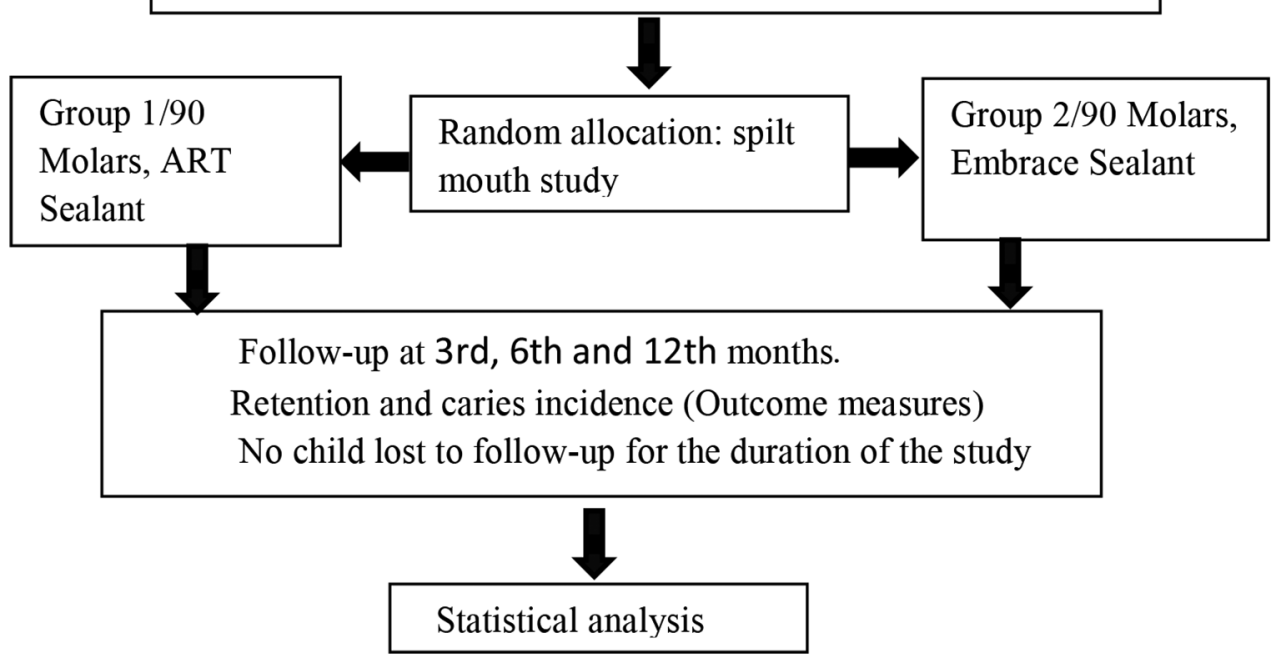

Fig. 1 Flowchart of the study procedure. ART, atraumatic restorative treatment.

Table 2 Comparison of modified Simonsen's scores between the two groups-Ketac Molar Easy Mix $(n=90)$ and Embrace WetBond $(n=90)$

\begin{tabular}{|c|c|c|c|c|c|c|c|}
\hline \multirow[t]{2}{*}{ Variables } & \multicolumn{5}{|c|}{ Modified Simonsen's Criteria (n [\%]) } & \multirow[t]{2}{*}{$X^{2}$-Value } & \multirow[t]{2}{*}{$p$-Value } \\
\hline & Score 0 & Score 1 & Score 2 & Score 3 & Score 4 & & \\
\hline \multicolumn{8}{|l|}{ 3rd month } \\
\hline GC Fuji Type IX & $59(65.6)$ & $25(27.8)$ & $4(4.4)$ & $2(2.2)$ & $0(0.0)$ & 2.340 & 0.673 \\
\hline WetBond sealant & $54(60.0)$ & $31(34.4)$ & $3(3.3)$ & $1(1.1)$ & $1(1.1)$ & & \\
\hline \multicolumn{8}{|l|}{ 6th month } \\
\hline GC Fuji Type IX & $20(22.2)$ & $42(46.7)$ & $10(11.1)$ & 17 (18.9) & $1(1.1)$ & 4.463 & 0.347 \\
\hline WetBond sealant & $25(27.8)$ & $39(43.3)$ & $12(13.3)$ & $10(11.1)$ & $4(4.4)$ & & \\
\hline \multicolumn{8}{|l|}{ 12th month } \\
\hline GC Fuji type IX & $7(7.8)$ & $27(30.0)$ & $21(23.3)$ & $28(31.1)$ & $7(7.8)$ & 2.877 & 0.579 \\
\hline WetBond sealant & $9(10.0)$ & $34(37.8)$ & $22(24.4)$ & $19(21.1)$ & $6(6.7)$ & & \\
\hline
\end{tabular}

resin-based sealants have an inherent drawback that limits its applicability in moisture-laden environments. This critical drawback prevents the usage of a hydrophobic resin-based sealant in a field setting where electrically powered equipment would be unavailable. Embrace WetBond sealant is a moisture-tolerant resin sealant, which is hydrophilic, water balanced, and is meant to adequately counter the shortcomings of conventional resin sealants.
Glass ionomer sealants have traditionally been an alternative to resin sealants due to its higher retentive properties. However, these sealants are not abrasive enough to resist occlusal masticatory forces. The ART sealants employing high viscosity glass ionomers with an elevated powder-liquid ratio are expected to fare better in the terms of retention.

This clinical trial had been formulated from a dental public health perspective. For sealants to be effective in 
community-based intervention programs, the application technique has to be far from being cumbersome, without the requirement of specialized electrically driven equipment. An inexperienced examiner was chosen, trained on the clinical aspects of these materials, and field tested in undesirable conditions, which would be a test of endurance for these sealants.

There are no standardized methods for assessing the adequacy of the sealed surfaces and therefore it poses significant challenges for an investigator willing to compare the outcomes of various clinical trials. A widely used criterion that was extensively used in large-scale public health services was proposed by Cvar and Ryge in 1971 and criticized due to subjective errors creeping in. ${ }^{29}$

Other criterion called the Color, Coverage, and Caries sealant evaluation was proposed by Deery et al in 2001, which records dental caries and describes the level of surface coverage..$^{30}$ A slightly modified United States Public Health Service criterion was also put to use by a few researchers. ${ }^{31}$ Simonsen's criterion by far has been used in most studies due to its ease of use, coupled with a high validity and reliability. ${ }^{27}$ This criterion was criticized for its inability to score dental caries and offers no explanation regarding partial loss of sealants. A modified Simonsen's criterion was employed to evaluate sealant surfaces in this trial as each code depicted the status of sealant coverage and the presence or absence of dentinal carious lesions.

In the field of retention, Embrace WetBond fared quite poorly with a retention rate of $10 \%$ complete retention at the end of 1 year. A comparison into the retention rate of embrace sealant, carried out by other investigators such as Bhatia et $\mathrm{al}^{22}$ (23\% complete retention/1 year), Schlueter et $\mathrm{al}^{25}$ (27\% complete retention/1 year) and Subramaniam et a ${ }^{32}$ ( $10 \%$ complete retention/ 1 year), has also found similar results when compared to the present study. However, contrasting results were seen in the studies carried out by Ratnaditya et $\mathrm{al}^{21}$ and Bhat et $\mathrm{al}^{23}$ (about $80 \%$ complete retention/1 year). A longitudinal study was carried out by Strassler and Donnell ${ }^{33}$ in a pediatric suburban practice, by sealing over 334 teeth using Embrace WetBond and found an astonishing retention rate of over $90 \%$ for a 4 to 6 years follow-up time.

A substantially low retention rate observed in this study has been contradictory to the previous in vitro study that demonstrated sealants to create bonds with high tensile strength. ${ }^{34}$ Nonetheless, the low retention rate could be attributed to the poor clinical handling of the Embrace WetBond sealant. The manufacturer recommends the tooth surface post-etching should be neither too dry for enamel desiccation to be seen nor too wet for the water droplets to be visualized. These clinical guidelines were to some extent difficult for an inexperienced clinician to fulfill especially in a field environment.

The moisture tolerance of the Embrace WetBond is due to the presence of hydrophilic monomers, which promotes the formation of bonds in the presence of moisture. However, these bonds increase water sorption and thus may enhance the solubility and a greater disintegration in an oral environment. ${ }^{35}$
Assuming that the poor retention of Embrace WetBond is caused due to its application in a field environment, previous studies $^{22,25}$ have observed poor retention rates with Embrace WetBond even in a carefully controlled clinical environment. On the contrary, some other studies ${ }^{21,23}$ have achieved higher retention rates, with the Embrace placed in similar conditions.

A bird's eye view of the above-mentioned results depicts a perplexing picture regarding the performance of Embrace WetBond, especially in the domain of retention. However, a few other factors strongly influence the longevity of the sealants. It has been advocated that sealant retention is better in the 6 to 7 years age group, attributable to the immature porous characteristic of enamel, thereby assisting sealant retention. ${ }^{36}$ On the other hand, when sealants were placed on fully erupted permanent molars in the age group between 9 and 11 years, increased stress induced by the masticatory forces caused poor retention. The poor retention of Embrace WetBond sealant could also be attributed to the inexperienced applicator, despite being trained adequately prior to the commencement of the trial.

It is quite apparent that Embrace WetBond has to be studied and field tested more extensively. The usage of a bonding agent was not required during the application, making the procedure less cumbersome and more cost-effective to be used in public health programs. Embrace WetBond, devoid of bisphenol GMA, negates the ill effects of exposure to bisphenol releasing materials that have been known to induce adverse health outcomes. ${ }^{37-39}$

The ART sealants in this clinical trial had a retention (complete and partial) of about $38 \%$ and has fared quite poor, when compared to the recent studies done by Holmgren et $\mathrm{al}^{40}$ (79\% cumulative survival rate at 2 years), Luengas-Quintero et $\mathrm{al}^{41}$ (48.8\% cumulative survival rate at 2 years), and Liu et $\mathrm{al}^{19}$ (52\% cumulative survival rate over 2 years). It has been a well-known fact that the experience of the operator is directly proportional to the retention of ART sealant.

Looking at the larger picture, there has been no significant difference between the sealants under study, at the end of 12 months, in terms of retention and caries prevention. A closer peek into the observed data at the designated time intervals revealed no clear-cut differences between the sealants, which is rather surprising. Embrace WetBond with its modified hydrophilic resin properties was expected to duplicate the superior retentive features of a resin sealant.

At the end of the follow-up period (12th month), 7.8\% of the teeth in the ART category had completely lost sealants with dentinal caries and $6.7 \%$ of the teeth in the Embrace category had completely lost sealants and presenting with evidence of dentinal caries. There was no statistically significant difference between these two groups in terms of caries prevention.

The results obtained, however, should be carefully analyzed in the light of current evidence. ART sealants use high viscosity glass ionomer cements, which possess an intrinsic property of fluoride release into its environment. ${ }^{42}$ This intrinsic nature of glass ionomer cements can provide a fluoride reservoir, thereby lowering the differences between 
the sealants in terms of fissure caries prevention. In addition, embrace has been known to release fluoride into the oral environment. We could not assess the impact of an elevated fluoride presence on the caries preventive benefits of the sealants. It has been widely acknowledged that an elevated presence of a free fluoride ion in the oral environment tips the balance in favor of remineralization. ${ }^{43}$

However, in this study, it was observed that the caries incidence among both groups was considerably higher with about $23 \%$ of the teeth in both groups presenting with evidence of caries at the end of the 12th month (Score 2). This is rather surprising as the follow-up duration was only for a year and such a substantial number of cavitated carious lesions were not expected to be seen for a disease considered to be chronic in nature.

The children enrolled in this clinical trial belonged to a low socioeconomic status and it is a known fact that socioeconomic factors in children have been a strong predictor of caries risk than it is for adults. ${ }^{44}$ It was also noted that these children were shifted to a new residential block by a nongovernmental organization in the recent past, thus leading to a lifestyle change and contributing to poor long-term maintenance of their oral hygiene. ${ }^{45}$ All these factors could have played a major role in spiking up the caries activity of these subjects.

An important drawback of the present study is the follow-up duration of 1 year, which can be considered as inadequate. The probability of finding a statistical difference between the performances of these sealants could have been higher, had the follow-up been for more than a year. Due to paucity of similar randomized controlled clinical trials, comparisons of the results could not be obtained.

It would be noteworthy to mention the conspicuous absence of oral prophylaxis prior to the application of sealants in this study as it could have played a role in downgrading the retentivity of the sealants. This can be considered as a major drawback of the study at the executional stage, as it was not feasible to carry out oral prophylaxis at an outreach facility. Investigators have recommended that the surface be devoid of soft debris, plaque for greater bond strength between the sealant and tooth surface, but controversy still persists about the effectiveness of this procedure. ${ }^{36}$

Resin-based sealants have been widely used in several clinical studies and are considered as a gold standard, which implies that the performance of newer preventive agents should be compared with the resin-based sealants as the baseline standard. Having said that, there is ample scope for ART sealants to enact a role as a positive control in this trial, simply because it has fared exceptionally well in terms of fissure caries prevention in limited clinical studies till date. ${ }^{19,20,46-48}$

In terms of handling characteristics, Embrace WetBond proved to be quite difficult for an inexperienced applicator to handle in this study. This has been primarily attributed to the inability of the applicator to maintain an etched tooth surface with a precise amount of moisture prior to the sealant placement and the need for requisite armamentarium to light cure the material. On the contrary, the use of ART sealants in the school environment was simple and straightforward.

\section{Conclusion}

Within the limitations of this study, it can be concluded that no significant difference was observed between these sealants either in the field of retention or fissure caries prevention. The data from this clinical trial tentatively suggests that ART sealants were less cumbersome to be employed in an outreach program. Embrace WetBond sealant needs to be thoroughly evaluated and field tested by experienced clinicians to ascertain its full potential as a hydrophilic resin-based sealant.

\section{Note}

This trial is registered with US National Library of Medicine (ClinicalTrials.gov NCT02716558, https://clinicaltrials. gov/). The manuscript has been read by all the authors and has been approved by each one of them. This is an honest effort by all the contributing authors of this manuscript.

\section{Funding}

None.

\section{Conflict of Interest}

None declared.

\section{Acknowledgments}

We sincerely thank the nonclinical staff of the dental institute for their active contribution toward the making of this manuscript.

\section{References}

1 Selwitz RH, Ismail AI, Pitts NB. Dental caries. Lancet 2007;369(9555):51-59

2 Young DA, Nový BB, Zeller GG, Hale R, Hart TC, Truelove EL; American Dental Association Council on Scientific Affairs. The American Dental Association Caries Classification System for clinical practice: a report of the American Dental Association Council on Scientific Affairs. J Am Dent Assoc 2015;146(2):79-86

3 Dye BA, Tan S, Smith V, et al. Trends in oral health status: United States, 1988-1994 and 1999-2004. Vital Health Stat 11 2007;(248):1-92

4 Dye BA, Thornton-Evans G, Li X, Iafolla TJ. Dental caries and sealant prevalence in children and adolescents in the United States, 2011-2012. NCHS Data Brief 2015;(191):1-8

5 Macek MD, Beltrán-Aguilar ED, Lockwood SA, Malvitz DM. Updated comparison of the caries susceptibility of various morphological types of permanent teeth. J Public Health Dent 2003;63(3):174-182

6 Wright JT, Crall JJ, Fontana M, et al. Evidence-based clinical practice guideline for the use of pit-and-fissure sealants: a report of the American Dental Association and the American Academy of Pediatric Dentistry. J Am Dent Assoc 2016;147(8):672-682.e12

7 Marthaler TM. Changes in dental caries 1953-2003. Caries Res 2004;38(3):173-181

8 Hopcraft MS, Morgan MV. Pattern of dental caries experience on tooth surfaces in an adult population. Community Dent Oral Epidemiol 2006;34(3):174-183

9 Ahovuo-Saloranta A, Forss $\mathrm{H}$, Walsh $\mathrm{T}$, et al. Sealants for preventing dental decay in the permanent teeth. Cochrane Database Syst Rev 2013; ( 3):CD001830

10 Evidence-based Clinical Practice Guideline for the Use of Pit-and-Fissure Sealants. Evidence-based clinical practice 
guideline for the use of pit-and-fissure sealants. Pediatr Dent 2016;38(6):263-279

11 Simonsen RJ, Neal RC. A review of the clinical application and performance of pit and fissure sealants. Aust Dent J 2011;56(Suppl 1):45-58

12 Ahovuo-Saloranta A, Forss H, Walsh T, Nordblad A, Mäkelä $\mathrm{M}$, Worthington HV. Pit and fissure sealants for preventing dental decay in permanent teeth. Cochrane Database Syst Rev 2017;7:CD001830

13 Hoffman I. A moisture tolerant, resin based pit and fissure sealant. Dental Tribune Dec; 2009 17A-18A. Industry Clinical

14 Pinkham JR, Casamassimo PS, Fields HW, Jr, McTigue DJ, Nowak A, Pediatric Dentistry: Infancy through Adolescence. 4th edition. Amsterdam, The Netherlands: Elsevier Health Sciences; 2005

15 Mickenautsch S, Yengopal V. Caries-preventive effect of glass ionomer and resin-based fissure sealants on permanent teeth: an update of systematic review evidence. BMC Res Notes 2011;4:22

16 Nagaraja UP, Kishore G. Glass ionomer cement - the different generations. Trends Biomater Artif Organs 2005;18(2):158-165

17 Frencken JE, Pilot T, Songpaisan Y, Phantumvanit P. Atraumatic restorative treatment (ART): rationale, technique, and development. J Public Health Dent 1996;56(3 Spec No):135-140, discussion 161-163

18 Frencken JE. The state-of-the-art of ART sealants. Dent Update 2014;41(2):119-120, 122-124

19 Liu BY, Xiao Y, Chu CH, Lo EC. Glass ionomer ART sealant and fluoride-releasing resin sealant in fissure caries prevention--results from a randomized clinical trial. BMC Oral Health 2014; $14: 54$

20 Zhang W, Chen X, Fan MW, Mulder J, Huysmans MC, Frencken JE. Do light cured ART conventional high-viscosity glass-ionomer sealants perform better than resin-composite sealants: a 4-year randomized clinical trial. Dent Mater 2014;30(5):487-492

21 Ratnaditya A, Kumar MM, Jogendra SS, Zabirunnisa M, Chaitanya RK, Chowdhary Kopuri RK. Clinical evaluation of hydrophobic and hydrophilic pit and fissure sealants-a two year follow-up study. J Young Pharm 2015;7(3):171

22 Bhatia MR, Patel AR, Shirol DD. Evaluation of two resin based fissure sealants: a comparative clinical study. J Indian Soc Pedod Prev Dent 2012;30(3):227-230

23 Bhat PK, Konde S, Raj SN, Kumar NC. Moisture-tolerant resin-based sealant: a boon. Contemp Clin Dent 2013;4(3):343-348

24 One-Year Clinical Success of Embrace Hydrophilic and Helioseal-F Hydrophobic Sealants in Permanent First Molars: A Clinical Trial. Nahid Askarizadeh, Haleh Heshmat, Nazanin Zangeneh. J Dent (Tehran) 2017;14(2):92-99

25 Schlueter N, Klimek J, Ganss C. Efficacy of a moisture-tolerant material for fissure sealing: a prospective randomised clinical trial. Clin Oral Investig 2013;17(3):711-716

26 Frencken JE, Pilot T, Van Amerongen E, Songpaisan Y, Pilot T, Manual for the Atraumatic Restorative Treatment Approach to Control Dental Caries. 3rd edition. Groningen: WHO Collaborating Centre for Oral Health Services Research; 1997

27 Simonsen RJ. Retention and effectiveness of dental sealant after 15 years. J Am Dent Assoc 1991;122(10):34-42

28 Oral Health Survey. Basic Methods. 4th ed. Geneva: World Health Organization; 1997:40

29 Cvar JF, Ryge G. Reprint of criteria for the clinical evaluation of dental restorative materials. 1971. Clin Oral Investig 2005;9(4):215-232
30 Deery C, Fyffe HE, Nugent ZJ, Nuttall NM, Pitts NB. A proposed method for assessing the quality of sealants--the CCC Sealant Evaluation System. Community Dent Oral Epidemiol 2001;29(2):83-91

31 Bayne SC, Schmalz G. Reprinting the classic article on USPHS evaluation methods for measuring the clinical research performance of restorative materials. Clin Oral Investig 2005;9(4):209-214

32 Subramaniam P, Jayasurya S, Babu KG. Evaluation of glass carbomer sealant and a moisture tolerant resin sealant-a comparative study. International Journal of Dental Science and Research. 2015;2(2-3):41-48

33 Strassler HE, O'Donnell JP. A unique moisture-tolerant, resin-based pit and fissure sealant: clinical technique and research results. Inside Dentistry 2008;4(9):108-110

34 Türkmen C, Durkan M, Cimilli H, Öksüz M. Tensile bond strength of indirect composites luted with three new self-adhesive resin cements to dentin. J Appl Oral Sci 2011;19(4):363-369

35 Ferracane JL. Hygroscopic and hydrolytic effects in dental polymer networks. Dent Mater 2006;22(3):211-222

36 Waggoner WF, Siegal M. Pit and fissure sealant application: updating the technique. J Am Dent Assoc 1996;127(3): 351-361, quiz 391-392

37 Kloukos D, Pandis N, Eliades T. In vivo bisphenol-a release from dental pit and fissure sealants: a systematic review. J Dent 2013;41(8):659-667

38 Michałowicz J. Bisphenol A--sources, toxicity and biotransformation. Environ Toxicol Pharmacol 2014;37(2):738-758

39 Jedeon K, De la Dure-Molla M, Brookes SJ, et al.Enamel defects reflect perinatal exposure to bisphenol A. Am J Pathol 2013;183(1):108-118

40 Holmgren CJ, Lo EC, Hu D. Glass ionomer ART sealants in Chinese school children-6-year results. J Dent 2013;41(9):764-770

41 Luengas-Quintero E, Frencken JE, Muñúzuri-Hernández JA, Mulder J. The atraumatic restorative treatment (ART) strategy in Mexico: two-years follow up of ART sealants and restorations. BMC Oral Health 2013;13:42

42 Williams B, Laxton L, Holt RD, Winter GB. Fissure sealants: a 4-year clinical trial comparing an experimental glass polyalkenoate cement with a bis glycidyl methacrylate resin used as fissure sealants. Br Dent J 1996;180(3):104-108

43 Featherstone JD. Prevention and reversal of dental caries: role of low level fluoride. Community Dent Oral Epidemiol 1999;27(1):31-40

44 National Institutes of Health. Diagnosis and Management of Dental Caries throughout Life. Bethesda, MD: National Institutes of Health; 2001

45 Fontana M, Zero DT. Assessing patients' caries risk. J Am Dent Assoc 2006;137(9):1231-1239

46 Beiruti N, Frencken JE, van't Hof MA, Taifour D, van Palenstein Helderman WH. Caries-preventive effect of a one-time application of composite resin and glass ionomer sealants after 5 years. Caries Res 2006;40(1):52-59

47 Chen X, Du MQ, Fan MW, Mulder J, Huysmans MC, Frencken JE. Caries-preventive effect of sealants produced with altered glass-ionomer materials, after 2 years. Dent Mater 2012;28(5):554-560

48 Oba AA, Dülgergil T, Sönmez IS, Doğan S. Comparison of caries prevention with glass ionomer and composite resin fissure sealants. J Formos Med Assoc 2009;108(11):844-848 